\title{
Non-invasive early diagnosis of oral squamous cell carcinoma using piezoelectric biosensor
}

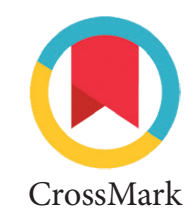

\author{
Irene E. Rieuwpassa, ${ }^{1 *}$ Sumintarti, ${ }^{2}$ Yu-Ri Kim, ${ }^{1}$ \\ Megatriani Matandung, ${ }^{1}$ Gabriel SA. Matongan ${ }^{3}$
}

\section{Abstract}

Objective: To develop a device capable of distinguishing Oral squamous cell from other types of lesion so early diagnosis can be made non-invasively.

Methods:The piezoelectric buzzer was coated with IgA EBV enzyme conjugate and antigen substance from Bionevan. The sensor was designed label free so further substance won't be needed. The body of the device was made from white acrylic with $4 \mathrm{~mm}$ thickness. The body was divided into 3 compartments each with different purpose. The main component consists of reagent coated piezoelectric buzzer and sensor. Both sensors are attached to Arduino UNO, breadboard, LIPO UBEC battery and LCD display network. The arduino UNO will read and process the difference between initial frequency and vibration frequency after the antigen were bind by the reagent, and shows the level of IgA in the LCD display.

Results: The device worked and manage to show the level of $\lg A$ and presence of $E B V$ in saliva but still need further improvement to make it more specific and valid

Conclusion: Piezoelectric biosensor have shown promise to make early non-invasive diagnosis for OSCC
${ }^{1}$ Departement of Oral Biology, Faculty of Dentistry, Hasanuddin University, Makassar, Indonesia ${ }^{2}$ Departement of Oral Medicine, Faculty of Dentistry, Hasanuddin University, Makassar, Indonesia ${ }^{3}$ Department of Electrical Engineering, Faculty of Engineering, Hasanuddin University, Makassar, Indonesia

*Correspondence to: Irene E. Rieuwpassa, Departement of Oral Biology, Faculty of Dentistry, Hasanuddin University, Makassar, Indonesia

drgirene@yahoo.com

Received: 20 June 2019

Revised: 1 July 2019

Accepted: 1 November 2020

Available Online: 1 December 2020

Keywords: Oral squamous cell carcinoma, Piezoelectric biosensor

Cite this Article: Rieuwpassa IE, Sumintarti, Kim Y, Matandung M, Matongan GSA. 2020. Non-invasive early diagnosis of oral squamous cell carcinoma using piezoelectric biosensor. Journal of Dentomaxillofacial Science 5(3): 146-149. D0I: 10.15562/jdmfs.v5i3.942

\section{Introduction}

Oral Squamous Cell Carcinoma (OSCC) is the manifestation of ulcerative lesion, which could be found in oral mucosa basal tissue. ${ }^{1}$ OSCC is the 6th most common found malignancy in the world; approximately 500.000 new cases of OSCC are found every year. ${ }^{2}$ OSCC has one of the highest mortality rate in comparison to other type of carcinoma due to difficulty in diagnosing it at early stage. Nearly all of OSCC advances from precancerous lesions such as leukoplakia and erythroplakia. Clinicians find it difficult to differentiate OSCC from precancerous lesion. ${ }^{3}$ Due to this difficulty, clinicians often are only able to diagnose OSCC when it has already reached advance lesion. Therefore, mortality rate for this cancer is high because treatment starts after the lesion has already advancedhence, there is an urgent need of marker to differentiate OSCC from precancerous lesion. These markers are vital to help increase the disease prognosis and life expectancy because it enables early diagnosis and treatment. ${ }^{4}$

Most clinical diagnosis requires additional examination such as biopsy for patients who could be diagnosed with OSCC, but this procedure is very invasive. Therefore, these days people seek for less invasive alternative to diagnose a disease, which is by using saliva. ${ }^{5}$ Like serum, saliva also contains hormones, antibodies, growth factors, enzymes, microbes and their products. Many of these constituents enter saliva through blood via passive diffusion, active transport or extracellular ultra filtration. Therefore, saliva can be seen in many cases as a reflection of the physiological function of the body. Some advantages of salivary testing for diagnosis are non-invasive, easy to use, inexpensive, safer to administer serum sampling, real-time diagnostic values, no need for trained medical staff, multiple samples can be obtained easily, collection and screening can be done at home, minimal risk of cross-contamination, more economical sampling and storage compared to serum, requires less manipulation during diagnostic procedures compared to serum, and commercial availability of screening assays. ${ }^{6}$ In addition saliva has been widely used in clinical research to detect diseases in oral cavity. ${ }^{7}$

Saliva contains many immunoglobulin (Ig) one of them is Immunoglobulin-A. IgA is the second most common Ig in serum and found extensively in secretions such as saliva, mucous, colostrum, and tears. It is, therefore, implicated in humoral immunity. Table 1 since IgA is associated with local immune response and saliva is in direct constant contact with oral lesions, salivary $\operatorname{IgA}$ is proposed to accurately reflect the changes caused by precancerous lesion and OSCC in the oral cavity. The increase in salivary $\operatorname{IgA}$ in OSCC is proposed to be due to increased local infection, increased antigenic inflammatory stimulus, increased local 
synthesis, and local host reaction to the disease. People with no lesion, precancerous lesion, and OSCC has different level of salivary. ${ }^{3}$ One of the way to measure IgA level is through Enzyme-Link Immunosorbent Assay (ELISA), but ELISA has drawbacks in its requirement of sample manipulation, time, costly equipment, and operation expertise. ${ }^{8,9}$ Therefore, a device that can measure Ig level with ease, handy, and cheap are necessary. Piezoelectric biosensor can be a solution to this problem. A biosensor is an analytical device, which converts a biological response into an electrical signal. The term 'biosensor' is often used to cover sensor devices used in order to determine the concentration of substances and other parameters of biological interest even where they do not utilize a biological system directly. Biosensors function by coupling a biological sensing element with a detector system using a transducer. ${ }^{10} \mathrm{~A}$ biosensor can be made as 'label-free' meaning it doesn't need any more reagents for the next usage once the piezoelectric sensor has been coated with reagent. Using biosensor as a mean of diagnosing OSCC by measuring level of salivary IgA can be very handy and easy.

\section{Material and Methods}

ELISA reagent (Bionevan China), Phosphate buffered saline (Biogear), distilled water, deionized water, pure ethanol, glutaraldehyde, bovine serum albumin (erybank), piezoelectric passive AC buzzer sensor, soldering iron, lead, arduino UNO, acrylic board, rainbow cable male-female, rainbow cable male-male, oscillator $10 \mathrm{MHz}$, battery, wind pump, LCD figure 1.

\section{Sensor Coating}

Sensor was washed before immobilization procedure starting. Sensor was consequently washed by deionized water and pure ethanol and let to dry in the next step, glutaraldehyde was solved in deionized water up to concentration $5 \% \mathrm{w} / \mathrm{w}$ and $50 \mu \mathrm{l}$ of the solution was spread over electrode placed into dark and wet box for at least 5 hours and then washed and dried again. Enzyme conjugate, substrate A and B (Bionevan) was spread over the sensor and let incubate in a dark and wet chamber overnight (12 hours). The surface was then washed

\section{Table 1 Salivary IgA level in different subjects}

\begin{tabular}{lc}
\hline Subject & Salivary lgA level $(\mathbf{m g} / \mathbf{d l})$ \\
\hline Healthy/normal & 75.51 \\
Precancerous lesion & 89.53 \\
OSCC & 94.47 \\
\hline
\end{tabular}

by a mild stream of PBS, dried and blocked by $5 \mathrm{mg} / \mathrm{ml}$ of bovine serum albumin (Erybank) for five hours in dark and wet chamber. The finished immunosensor was washed by a mild stream of by PBS with $0.1 \%(\mathrm{w} / \mathrm{w})$ Tween 20 prior to use. The electrodes were stored in dry state in a paper box at $4^{\circ} \mathrm{C}$ until use in the experiments.

\section{Device body}

The device body was made using white colored $4 \mathrm{~mm}$ thick acrylic. The body is comprised of 3 compartments; the first compartment was designed to store the main components, the second compartment to store the battery and arduino network, and the third to store the mini vacuum. The acrylic was cut into smaller pieces and glued together. Small holes was made for cables, switch, saliva holes and wind holes.

\section{Main Components Assembling}

The main component consists of IgA EBV reagent coated piezoelectric buzzer also piezoelectric sensor. Before saliva was dripped above the buzzer, sensor will read the buzzer's initial vibration frequency, after the saliva has been dripped above the sensor, reagent will bind the specific antigen and increase the buzzer's weight thus slowing it vibration's frequency. The shift in buzzer's vibration will be processed by arduino.

\section{Components network assembling}

The network consist of arduino UNO, breadboard, LIP UBEC 5V battery, and LCD display. The network were combined with the main components to process the data collected from the piezoelectric sensor. The results were processed by the arduino UNO system and then the results will be shown on the LCD Display figure 2.

\section{Mini Vacuum and hole drilling}

This component is used to suck the saliva after use. The holes were made using drills; holes made were used for cables, saliva, and wind holes.

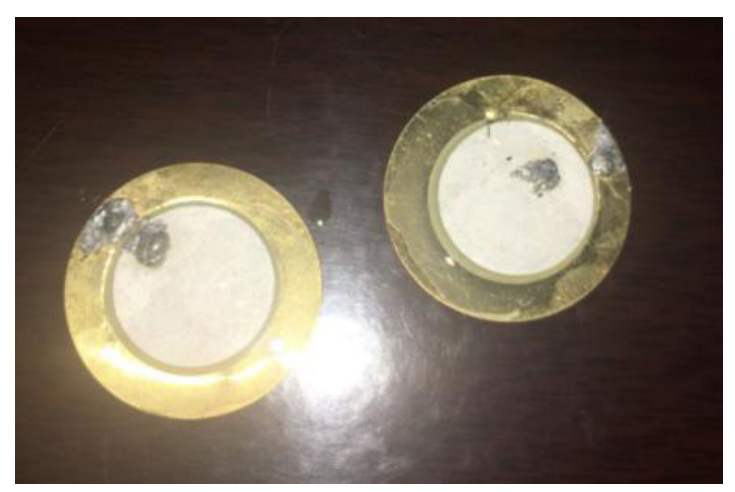

Figure 1 Coated piezoelectric 


\section{Results}

Figure 3 the immunoglobulin biosensor device was successfully assembled. The prepared immunosensor were tested for IgA level on normal and apthousstomatitis patients. After $2 \mathrm{ml}$ of saliva was collected from each category, the saliva was then poured on the hole which directly falls on the sensor. The buzzer then vibrates the sensor and the difference in frequency was counted and processed on the arduino UNO. The data collected will be shown on the monitor and to ease the use, the monitor will only present negative or positive according to the range of IgA level counted by the arduino UNO. After each sample IgA level is identified, the

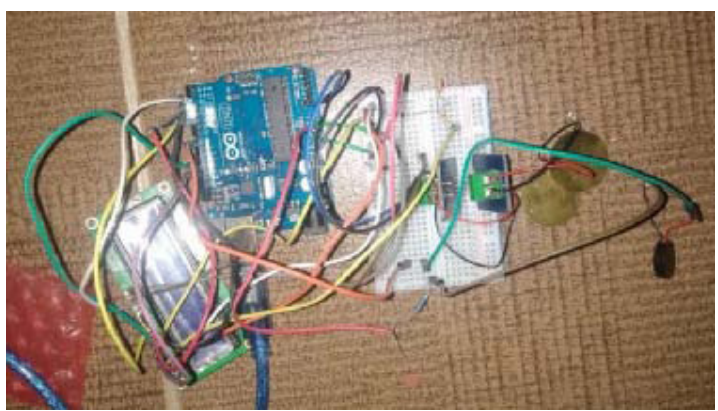

Figure 2 Sensor assembling

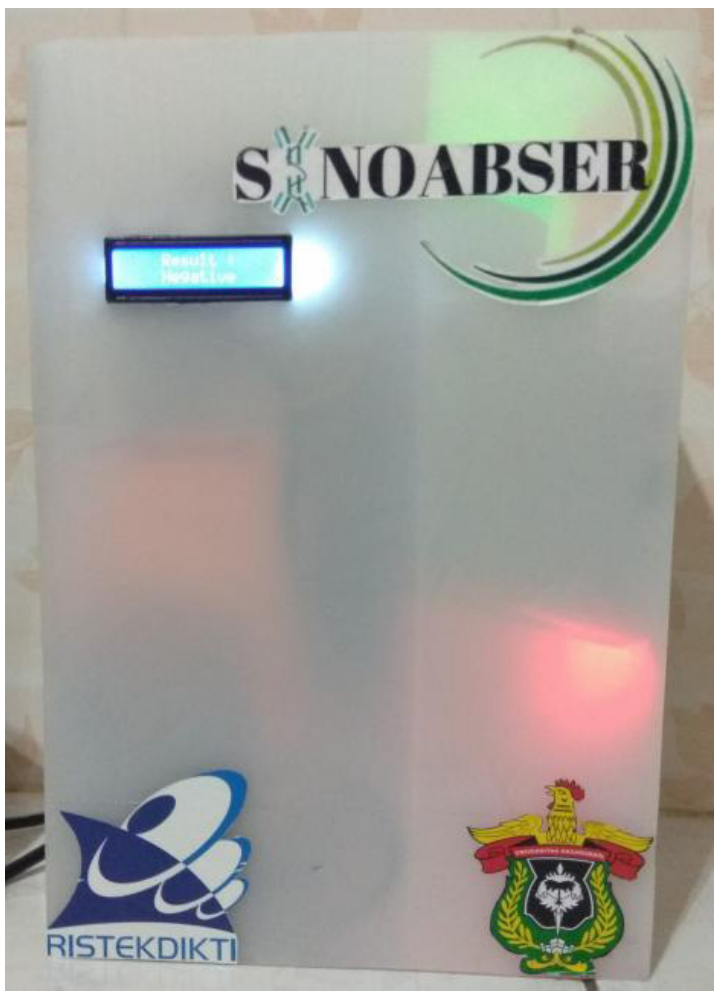

Figure 3 Immunoglobulin a biosensor device sensor is washed with buffered solution to prevent bias in the next IgA level count. The device worked well seeing that it didn't show positive results in patients with stomatitis, which is another type of white lesion.

\section{Discussion}

This device was made using piezoelectric biosensor principle. Piezoelectric is a physical phenomenon that leads to the ability of certain materials to produce electrical current when given mechanical pressure. ${ }^{11}$ Physicochemical transducer alters physical or chemical changes by antigen and antibody reaction into electrical current. Because this device uses specific antigen, hence this sensor is sensitive only to one certain antibody, in this case Immunoglobulin A in human saliva. This device work by the vibrating the sensor that has been coated before and measure the difference in frequencies between the vibration before and after antibody binds with antigen. ${ }^{12}$ Antigen and antibody (IgA) binds when the saliva was poured on top of the sensor. Before saliva was poured on top of the sensor, the sensor was first vibrated using oscillator and the frequency was measured, after the saliva was poured and IgA was bound the frequency changed due to the weighed added on top of the coat, the difference in frequency is then calculated by Arduino and the results will appear on the screen whether the subject are tested positive or negative.

In order to simply test the sensitivity of the device, to investigate whether the device will test positive on any subject, the device was tested on both normal and patient with oral lesion that could possibly have an increase in IgA level namely apthous stomatitis. ${ }^{13}$ Some studies have shown that patient with stomatitis has higher IgA level than normal patient without the lesion because IgA plays an important role in protection against infection. ${ }^{13,14}$ Henceforth the device was tested on stomatitis subject. The device shows negative results for both stomatitis subjects because the number of salivary IgA in stomatitis patient is still lower. ${ }^{14}$ Further studies need to be conducted to validate the data, calibration, and to make the sensor more specific.

\section{Conclusion}

Piezoelectric biosensor have shown promise to make early non-invasive diagnosis for OSCC. 


\section{Acknowledgment}

The authors would like to say our deepest gratitude to Hasanuddin University Faculty of Dentistry and Electrical Engineering for all the support provided during this research.

\section{Conflict of Interest}

The authors report no conflict of interest.

\section{References}

1. Gleber-Netto FM. Salivary biomarkers for detection of oral squamous cell carcinoma in a Taiwanese population. Clin Cancer Res 2016;1:OF1.

2. Sathiyasekar AC, Chandrasekar P, Pakash A, et al.Overview of immunology of oral squamous cell carcinoma. J Pharm Bioallied Sci 2016;8: S8.

3. Ajila V, Shetty V, Babu S, et al. Immunoglobulin a in oral potentially malignant disorders and oral squamous cell carcinoma. J Med Sci 2017;37:195-197.

4. Radhika T, Jeddy S, Nithya RM, et al. Salivary biomarkers in oral squamous cell carcinoma - An insight. J Oral Bio Craniofacial Res 2016;6: S51.

5. Malon RSP, Sadir D, Balakrishnan M, et al. Saliva-based biosensors: noninvasive monitoring tool for clinical diagnostics. BioMed Res Int 2014;1:1,12

6. Javaid MA, Ahmed AS, Durand R, et al. Saliva as a diagnostic tool for oral and systemic diseases. J Oral Bio Craniofacial Res 2016;6: 66-67.
7. Wulansari DP, Arsyad A, Rieuwpassa IE, Hamrun N, Hasyim R. Analysis of genotoxic and cytotoxic effects of oral mucosa in smokers and non-smokers after exposed to digital intraoral radiography. J Dentomaxillofac Sci 2019;4(3):159.

8. Liu Y, Li Y, Chang R, et al. Identification of proteinaceous binders in Ancient Tripitaka by the use of an enzymelinked immunosorbent assay. Anal Sci 2016;32: 735

9. Thiha A, Ibrahim F. A Colorimetric Enzyme-Linked Immunosorbent Assay (ELISA) Detection Platform for a Point-of-Care Dengue Detection System on a Lab-onCompact-Disc. Sensors 2015;15: 11431.

10. Malhotra S, Verma A, Tyagi N, et al. Biosensors: principle, types, and applications. IJIARIIE 2017;3: 3639.

11. Pohanka M. Overview of piezoelectric biosensors, immunosensors and DNA sensors and their applications. Materials (Basel) 2018;11: 1-4.

12. Pohanka M. The piezoelectric biosensors: principles, applications, a review. Int J Electrochem Sci 2017;12: 496-497.

13. Saluja RM Kale A, Hallikerimath S, Determination of levels of salivary IgA subclasses in patients with minor recurrent apthous ulcer. J Oral Maxillofac Pathol 2012;16: 50.

14. Mohammad R, Halboub E, Mashlah A, et al. Levels of salivary IgA in patients with minor recurrent apthous stomatitis: a matched case-control study. Clin Oral Investig 2013;17: 975 .

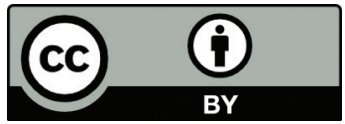

This work is licensed under a Creative Commons Attribution 